\title{
Silicon Photonics for Coherent Terahertz Generation and Detection
}

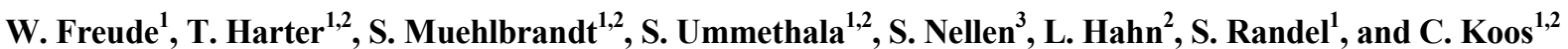 \\ ${ }^{1}$ Institute of Photonics and Quantum Electronics (IPQ), ${ }^{2}$ Institute of Microstructure Technology (IMT), \\ Karlsruhe Institute of Technology (KIT), Germany \\ ${ }^{3}$ Fraunhofer Institute for Telecommunications, Heinrich Hertz Institute (HHI), Berlin \\ Tel: +49721 608-42492, Fax: +49721608-9-42492,e-mail: w.freude@kit.edu
}

\begin{abstract}
Silicon-plasmonic internal photoemission devices can act as photomixers for generating terahertz frequency carriers (T-waves) for transmitters ( $\mathrm{Tx}$ ), or they function as receivers ( $\mathrm{Rx}$ ) for coherently downconverting $\mathrm{T}$ wave signals to the baseband. In a first demonstration, we monolithically integrate a Tx and a Rx on a silicon chip and operate them in a carrier frequency range up to $1 \mathrm{THz}$. With a co-integrated transmission line both components can be connected.
\end{abstract}

Keywords: THz waves, photomixing, internal photoemission, plasmonics, homodyne reception.

\section{INTRODUCTION}

In recent years, the use of terahertz carriers (T-waves) for wireless data transmission has become possible due to the availability of narrowband sources and suitable receivers. Both, optoelectronic signal processing and active microwave monolithic integrated circuits (MMIC) can be employed on the transmitter (Tx) and on the receiver $(\mathrm{Rx})$ side [1]. However, the fabrication of optoelectronic circuits relies on a complex processing of III-V semiconductor substrates [2] [3], and the manufacture of MMIC requires sophisticated state-of-the-art metamorphic high electron mobility transistor (mHEMT) technology [4] [5] based on GaAs. This prevents an easy cointegration of $\mathrm{T}$-wave $\mathrm{Tx}$ and $\mathrm{Rx}$ with digital signal processing circuits realized in CMOS technology on the silicon platform.

Here we report how these limitations can be overcome by a plasmonic internal-photoemission device (PIPED) [6] [7], which is fabricated on the silicon photonic platform with a footprint of only $1 \mu \mathrm{m}^{2}$. We integrate a PIPED Tx and a Rx on a common silicon chip and demonstrate $\mathrm{T}$-wave signal generation and coherent detection at carrier frequencies up to $1 \mathrm{THz}$ [8]. Further, we connect a PIPED Tx and a Rx by a simultaneously integrated transmission line with a view towards lab-on-chip applications [8].

\section{STRUCTURE AND FUNCTIONING OF PLASMONIC INTERNAL-PHOTOEMISSION DEVICE}

Figure 1(a) shows a schematic of a PIPED. Light with a vacuum wavelength $1550 \mathrm{~nm}$ (photon energy $\hbar \omega=0.8 \mathrm{eV}, f=\omega /(2 \pi)=193.41 \mathrm{THz}$ ) is launched into a $300 \mathrm{~nm}$ high and $400 \mathrm{~nm}$ wide silicon-on-insulator (SOI) strip waveguide (Si WG), and operated in quasi-TE polarization (dominant transverse electric field parallel to the substrate). A $550 \mathrm{~nm}$ long tapered waveguide section converts the strip WG mode into a surface plasmon polariton (SPP), which is guided in a metal-semiconductor-metal (MSM) junction. The junction consists of a $\mathrm{Si}$ core which is $300 \mathrm{~nm}$ high and typically $w=75 \mathrm{~nm}$ wide on top. It is covered with gold (Au) on one side, and with titanium (Ti) on the other side. Between these metal electrodes a bias voltage $U$ is applied, counted positive from the Au to the Ti electrode. The external current $I$ is counted positive in the direction towards the Au electrode. The MSM junction is schematically shown in Fig. 1(b). The metal covers have a thickness of $t=40 \mathrm{~nm}$. The junction length, typically $L \geq 1 \mu \mathrm{m}$, is uncritical, because the SPP absorption length is smaller than $1 \mu \mathrm{m}$.
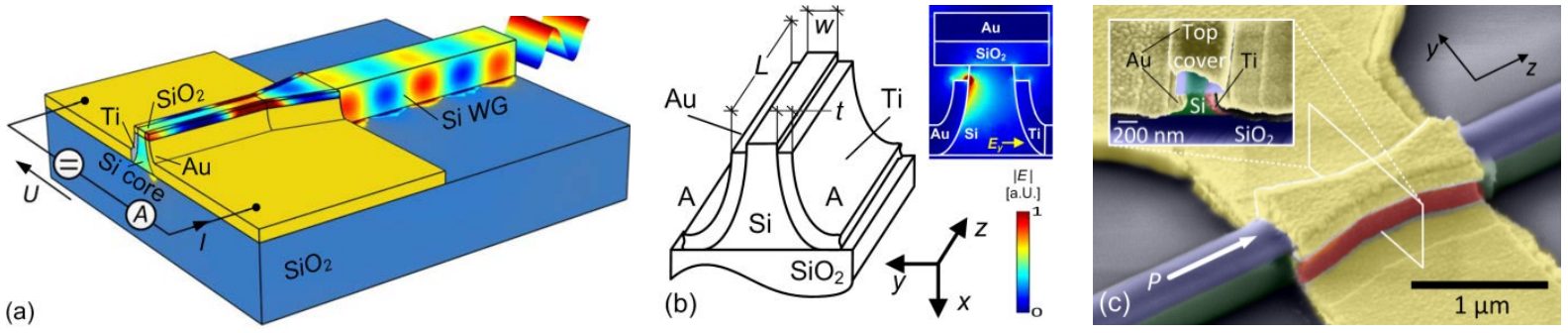

Fig. 1. Plasmonic internal photoemission device (PIPED) coupled to a silicon optical waveguide. (a) Half of a PIPED. Light with a wavelength of $1550 \mathrm{~nm}$ enters the silicon strip waveguide ( $\left.\mathrm{Si} \mathrm{WG)} \mathrm{(400} \mathrm{nm} \mathrm{width)} \mathrm{supported} \mathrm{by} \mathrm{a} \mathrm{thick} \mathrm{silicon-oxide}(\mathrm{SiO})_{2}\right)$ layer. A $550 \mathrm{~nm}$ long tapered Si WG section acts as a photonic-to-plasmonic converter. The resulting surface plasmon polariton (SPP) is guided by a goldsilicon $(\mathrm{Au})$ and a silicon-titanium (Ti) interface, but is absorbed mainly at the Ti side. The SPP absorption length in the plasmonic waveguide is smaller than $1 \mu \mathrm{m}$, therefore the length of the PIPED junction is uncritical. The Au-Si-Ti junction is biased with the voltage $U$. The resulting current $I$ is counted positive when flowing into the Au electrode. (b) Schematic drawing of MSM junction. A Si core with a height of $300 \mathrm{~nm}$ and a length of $L=(1 \ldots 20) \mu \mathrm{m}$ is sandwiched between two metallic layers (Au, Ti) with a thickness $t=40 \mathrm{~nm}$. The core has a top width $w=(75 \ldots 200) \mathrm{nm}$. An $\mathrm{SiO}_{2}$ hard mask and an Au top cover remain after processing as is to be seen in the inset. The electric field $E_{y}$ in the $y$-direction dominates. The computed modulus of the optical electric field strength is colour-coded. (c) Top view. Falsecoloured scanning electron micrograph (SEM) with silicon photonic strip waveguide, incident optical power $P$, and photonic-to-plasmonic mode converter. Inset: SEM of the cross-section. [Fig. 1(a,b,c) modified from [7] (C) 2017 IEEE, [6] (C) 2017 OSA, and [8] C) 2018 SNPG] 
Non-equilibrium, $U>\varphi_{\mathrm{bi}}$

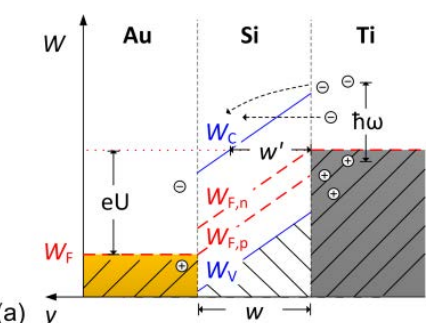

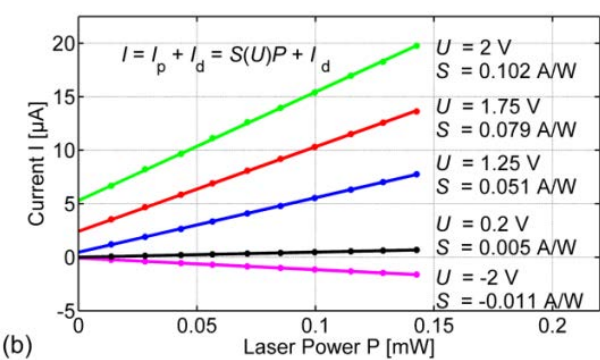

(b)

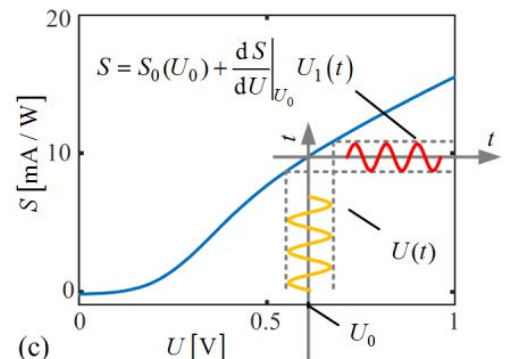

Fig. 2. Energy band diagram and current-voltage characteristics of the Au-Si-Ti junction. (a) Energy $W$, silicon conduction and valence band edges $W_{C}$ and $W_{V}$, Fermi level $W_{F}$, quasi Fermi levels $W_{\mathrm{F}, \mathrm{n}}$ and $W_{\mathrm{F}, \mathrm{p}}$ for electrons and holes, Schottky barrier heights for electrons $\Phi_{\mathrm{Au}}=0.82 \mathrm{eV}$ and $\Phi_{\mathrm{Ti}}=0.62 \mathrm{eV}$, built-in potential $\varphi_{\mathrm{bi}}=0.2 \mathrm{~V}$, photon energy $\hbar \omega=0.8 \mathrm{eV}(\lambda=1.55 \mu \mathrm{m})$, current $I$ (counted positive from $\mathrm{Ti}$ to $\mathrm{Au}$ ), bias voltage $U$ (counted positive from Au to Ti), junction width $w=75 \mathrm{~nm}$, junction length $L=5 \mu \mathrm{m}$. Non-equilibrium, forward bias voltage $U>\varphi_{\mathrm{bi}}$. The bands tilt strongly. The Fermi level splits into quasi Fermi levels for electrons and holes (nearly parallel to the band edges), and drop and rise sharply at the Au and at the Ti electrode, respectively. The barrier width $w$ reduces to an effective width $w^{\prime}$, and with increasing forward bias the photo-generated hot carriers in Ti are more likely to either overcome the Schottky barrier or to tunnel through. (b) Current-voltage characteristics. The total current $I=I_{\mathrm{p}}+I_{\mathrm{d}}$ has two contributions: First, the photocurrent $I_{\mathrm{p}}=S P$, which is a function of the voltage-dependent sensitivity $S$ and of the optical power $P$ (here, $P=310 \mu \mathrm{W}$, measured at the mode converter input), and second, the dark current $I_{\mathrm{d}}$. For a forward bias $U=3.25 \mathrm{~V}$, the photocurrent $I_{\mathrm{p}}=38 \mu \mathrm{A}$ is positive, and the sensitivity reaches a record value of $S=0.12 \mathrm{~A} / \mathrm{W}$. (c) Bias-dependent sensitivity $S(U)$ and linearization at operating point $U=U_{0}$. [Fig. 2(a,b,c) modified from [7] (C) 2017 IEEE, [6] C 2016 OSA, and [8] C 2018 SNPG]

The inset shows the magnitude of the electric field in the junction when excited with a quasi-TE mode. The $\mathrm{SiO}_{2}$ hard mask on top of the core and its Au top cover are remnants of the PIPED fabrication. A false-coloured scanning-electron micrograph (SEM) of the MSM junction, the photonic-to-plasmonic mode converters, and the two connecting photonic strip waveguides is displayed in Fig. 1(c). The active portion of the PIPED requires an area of less than $1 \mu \mathrm{m}^{2}$. The SPP is absorbed mainly at the Si-Ti interface, because for Ti the magnitude of the imaginary part of the complex relative dielectric constant is about five times larger than for Au. If a sufficiently large forward bias voltage $U$ is applied, the photoelectrons generated in the Ti layer move across the silicon core towards the Au electrode.

The energy band diagram of the MSM junction in thermal equilibrium $(U=0)$ is characterized by a constant Fermi energy $W_{F}$ throughout the junction. The conduction and valence band edges in the weakly $p$-doped $\left(N_{A} \approx 10^{15} \mathrm{~cm}^{-3}\right)$ Si core are marked with $W_{C}$ and $W_{V}$, respectively. The Schottky barrier height for electrons at the Au-Si interface $\Phi_{\mathrm{Au}}=0.82 \mathrm{eV}$ is by $30 \%$ larger than the Si-Ti potential barrier $\Phi_{\mathrm{Ti}}=0.62 \mathrm{eV}$. The SPP that propagate in the $\mathrm{Si}$ core generate, within a few $\mathrm{nm}$ from the interface, hot electrons in the metal electrodes with a maximum energy of $\hbar \omega=0.8 \mathrm{eV}$ above the respective Fermi levels. These electrons can cross the potential barriers by thermionic emission or by tunneling, a process called internal photoemission [9]. A built-in potential of $\varphi_{\mathrm{bi}}=\left(\Phi_{\mathrm{Au}, \mathrm{e}}-\Phi_{\mathrm{Ti}, \mathrm{e}}\right) / \mathrm{e}=0.2 \mathrm{~V}$ results, and no significant current flow can be measured.

If a bias $U>\varphi_{\mathrm{bi}}$ is chosen, Fig. 2(a), the Au electrode becomes positive with respect to the Ti electrode, and the Fermi energy in Au becomes smaller than in thermal equilibrium. Because the depletion zone in the weakly doped silicon core would be much larger than the actual core width, the bias voltage $U$ leads to a virtually constant electric field in the core, and the band edges remain straight but tilted. The Fermi level splits into quasi Fermi levels for electrons $\left(W_{F, n}\right)$ and holes $\left(W_{F, p}\right)$ which run nearly parallel to the band edges, and drop and rise sharply at the $\mathrm{Au}$ and at the Ti electrode, respectively. The barrier width $w^{\prime}$ at the Si-Ti interface reduces, and photo-generated carriers are more likely to cross the potential barrier.

For a bias of $U=1 \mathrm{~V}(U=3 \mathrm{~V})$ and a Si core width $w=75 \mathrm{~nm}$, the potential barrier width for electrons at Fermi level energy in Ti reduces to $w^{\prime}=58 \mathrm{~nm}\left(w^{\prime}=17 \mathrm{~nm}\right)$. For hot electrons, the effective potential barrier becomes even smaller, resulting in a significantly larger sensitivity than would be expected from plain internal photoemission. The large electric field in the silicon core accelerates the carriers up to their saturation velocity $100 \mathrm{~nm} / \mathrm{ps}=10^{7} \mathrm{~cm} / \mathrm{s}$, leading to a maximum transit time of $750 \mathrm{fs}$. The capacitance for a MSM junction with height $275 \mathrm{~nm}$, width $w=75 \mathrm{~nm}$ and length $L=5 \mu \mathrm{m}$ is estimated to be smaller than $C=2 \mathrm{fF}$.

The current vs. laser power characteristics of a PIPED are displayed in Fig. 2(b). The measured current $I=I_{\mathrm{p}}+I_{\mathrm{d}}$ consists of a photocurrent $I_{\mathrm{p}}=S P$ (sensitivity $S$, optical power $P$ ) and of a dark current $I_{\mathrm{d}}$. Both currents depend on the external bias voltage $U$. With a positive bias $U>\varphi_{\mathrm{bi}}$ and for a fixed illumination $P=310 \mu \mathrm{W}$ at the mode converter input, the total current $I$ increases steeply for $\varphi_{\mathrm{bi}}<U<2 \mathrm{~V}$ because of the increasing tunneling probability at the Si-Ti potential barrier. Therefore the sensitivity $S(U)=I_{\mathrm{p}} / P$ depends strongly on the barrier width $w^{\prime}$, see Fig. 2(b). For $U>1 \mathrm{~V}$ the sensitivity increases exponential-like. At a bias of $U=3.25 \mathrm{~V}$ the sensitivity amounts to $S(3.25 \mathrm{~V})=0.12 \mathrm{~A} / \mathrm{W}$, more than six times larger than for other internal photoemission detectors [10] [11].

The current increases strictly linearly with the optical power up to a value of at least $P=143 \mu \mathrm{W}$, measured at the input of the photonic-to-plasmonic mode converter. This proves that two-photon absorption in silicon is insignificant. Avalanche multiplication contributes only moderately even at higher field strengths: With a $w=75 \mathrm{~nm}$ wide junction and at a bias of $U=1 \mathrm{~V}$ (or $U=3.25 \mathrm{~V}$ ) the electric field strength and the Si ioniza- 
tion coefficient [12] are $E=U / w=13 \mathrm{~V} / \mu \mathrm{m}$ and $\alpha_{\mathrm{i}}=5 \times 10^{-4} \mu \mathrm{m}^{-1}$ (or $E=43 \mathrm{~V} / \mu \mathrm{m}$ and $\alpha_{\mathrm{i}}=6.7 \mu \mathrm{m}^{-1}$ ), respectively, which leads to an avalanche multiplication factor of $M_{0}=\exp \left(\alpha_{\mathrm{i}} w\right)=1$ (or $\left.M_{0}=1.6\right)$.

\section{T-WAVE GENERATION AND COHERENT DOWN-CONVERSION}

A PIPED can be used to generate and to coherently receive a T-wave. The basic mechanism of T-wave generation is photomixing which can be achieved with any photon-absorbing detector, and is not a peculiarity of the PIPED. Consider the superposition $E(t)$ of two optical carriers (O-waves) $E_{\mathrm{a}, \mathrm{b}}(t)$ with electric field amplitudes $\hat{E}_{a, b}$ and frequencies $f=\omega /(2 \pi)$ and $f+f_{\mathrm{THz}}$. The superposition is characterized by an optical power $P(t)$ which oscillates with the difference frequency $f_{\mathrm{THz}}$,

$$
\begin{aligned}
E(t) & =\hat{E}_{a} \cos (\omega t)+\hat{E}_{b} \cos \left[\left(\omega+\omega_{\mathrm{THz}}\right) t\right]=\left[\hat{E}_{a}+\hat{E}_{b} \cos \left(\omega_{\mathrm{THz}} t\right)\right] \cos (\omega t)-\hat{E}_{b} \sin \left(\omega_{\mathrm{THz}} t\right) \sin (\omega t), \\
P(t) & =\left\langle E^{2}(t)\right\rangle_{2 \pi / \omega}=\underbrace{\frac{1}{2} \hat{E}_{a}^{2}+\frac{1}{2} \hat{E}_{b}^{2}}_{P_{0}}+\underbrace{\hat{E}_{a} \hat{E}_{b}}_{\hat{P}_{1}} \cos \left(\omega_{\mathrm{THz}} t\right), \quad I(t)=S P(t)=I_{0}+\hat{I}_{1} \cos \left(\omega_{\mathrm{THz}} t\right) .
\end{aligned}
$$

The terms $\cos ^{2}(\omega t), \sin ^{2}(\omega t)$ and $\cos (\omega t) \sin (\omega t)=\frac{1}{2} \sin (2 \omega t)$ resulting from the squaring operation are averaged over an optical period $2 \pi / \omega$ as is required by the definition of power, and do therefore not contribute. The rate of arriving photons with energy $\hbar \omega$ depends on the power $P(t)=P_{0}+\hat{P}_{1} \cos \left(\omega_{\mathrm{THz}} t\right)$. If this power is incident on a photodetector, its output current $I(t)=S P(t)$ also oscillates with frequency $f_{\mathrm{THz}}$ and may be used to excite an antenna for wireless transmission. The difference frequency $f_{\mathrm{THz}}$ between the laser fields $E_{\mathrm{a}, \mathrm{b}}(t)$ can be easily tuned to lie in the THz range. Data are transferred to the T-wave carrier by electrooptically modulating either $E_{\mathrm{a}}(t)$ or $E_{\mathrm{b}}(t)$ by amplitude and/or by phase.

In contrast to a usual pin-photodetector with a constant sensitivity $S=S_{0}$, a PIPED can also coherently receive a T-wave as explained in the following. Figure 2(c) displays a measured $S(U)$-characteristic for an average bias voltage $U_{0}$. If this bias voltage $U(t)$ as received by a T-wave antenna varies sinusoidally having a small amplitude $U_{1}$ and a frequency $f_{\mathrm{THz}}$, then the PIPED phototocurrent $I^{\prime}(t)$ varies sinusoidally with the same frequency $f_{\mathrm{THz}}$, given that the optical input power $P^{\prime}(t)=P_{0}^{\prime}$ is constant. With a sinusoidally changing optical power as in Eq. (1), but with a frequency $f_{\mathrm{THz}}^{\prime}$, the electronic baseband current (E-wave) $I_{\mathrm{BB}}(t)$ results from the product of two sinusoidals with frequencies $f_{\mathrm{THz}}$ and $f_{\mathrm{THz}}^{\prime}$, and therefore varies sinusoidally at an intermediate frequency $\left|f_{\mathrm{THz}}-f_{\mathrm{THz}}^{\prime}\right|$ with an amplitude $I_{\mathrm{BB}, 1}$. This means that the PIPED acts as a heterodyne mixer, down-converting an incoming T-wave, which could be modulated by amplitude and phase, with the help of an optically generated local oscillator (LO),

$$
\begin{aligned}
I^{\prime}(t) & =S(U(t)) P^{\prime}(t), \quad U(t)=U_{0}+\hat{U}_{1} \cos \left(\omega_{\mathrm{THz}} t\right), \quad P^{\prime}(t)=P_{0}^{\prime}+\hat{P}_{1}^{\prime} \cos \left(\omega_{\mathrm{THz}}^{\prime} t\right), \\
I_{\mathrm{BB}, 1}(t) & =\hat{I}_{\mathrm{BB}, 1} \cos \left[\left(\omega_{\mathrm{THz}}-\omega_{\mathrm{THz}}^{\prime}\right) t\right], \quad \hat{I}_{\mathrm{BB}, 1}=\left.\frac{1}{2} \frac{\mathrm{d} S}{\mathrm{~d} U}\right|_{U_{0}} \hat{P}_{1}^{\prime} \hat{U}_{1} .
\end{aligned}
$$

If $\mathrm{LO}$ and incoming $\mathrm{T}$-wave carrier are phase-locked, i. e., if $f_{\mathrm{THz}}^{\prime}=f_{\mathrm{THz}}$, a homodyne receiver is established.

Figure 3(a) shows the schematic of an $\mathrm{O}$-wave-to-T-wave $(\mathrm{O} / \mathrm{T})$ converter, functioning as a $\mathrm{T}$-wave transmitter (Tx) according to Eq. (1), and a coherent T-wave-to-E-wave converter as a coherent receiver (Rx) according
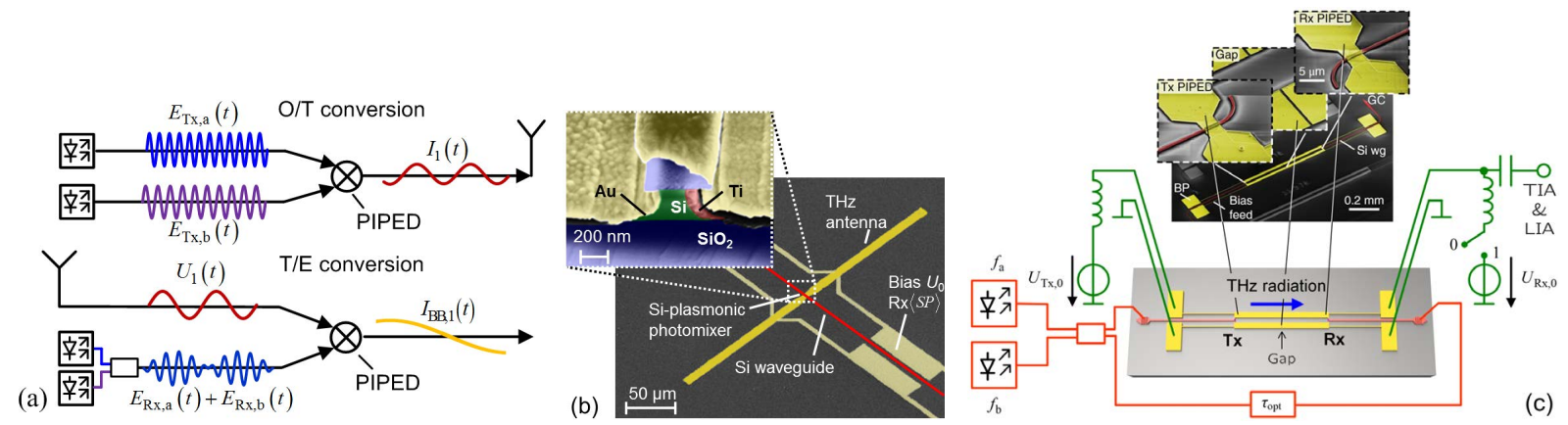

Fig. 3. Experimental setup for generating and coherently receiving T-waves with a PIPED. (a) Top: T-wave generation by photomixing $\left(\mathrm{O} / \mathrm{T}\right.$ conversion). The superposition of optical fields $E_{\mathrm{Tx}, \mathrm{a}}(t), E_{\mathrm{Tx}, \mathrm{b}}(t)$ generates a T-wave current $I_{1}(t)$ which feeds an antenna. (a) Bottom: Coherent T-wave reception (T/E conversion). The sensitivity $S\left(U_{1}(t)\right)$ is controlled by the received T-wave. The superposition of optical fields $E_{\mathrm{Rx}, \mathrm{a}}(t)+E_{\mathrm{Rx}, \mathrm{b}}(t)$ acts as a local T-wave oscillator, which feeds the PIPED and converts $U_{1}(t)$ to an intermediatefrequency (heterodyne Rx) or to a baseband current (homodyne Rx) $I_{\mathrm{BB}, 1}(t)$. (b) False-coloured scanning electron microscopy (SEM) image of an $\mathrm{O} / \mathrm{T}$ or T/E converter coupled to a dipole antenna. The PIPED is fed with a superposition of optical fields $E(t)=E_{\mathrm{a}}(t)+E_{\mathrm{b}}(t)$ according to Eq. (1) through silicon-photonic strip waveguides (red), and biased via a low-pass network (T-wave chokes) connected to highimpedance points of the antenna. (c) Schematic of a silicon-integrated T-wave homodyne system comprising a Tx, a coplanar transmission line with a maximum length of $1 \mathrm{~mm}$, and an Rx. Light is coupled to the Tx and Rx PIPED by silicon waveguides (red) and grating couplers (GC, see inset). An optical delay line $\tau_{\mathrm{opt}}$ adjusts the phase difference between the Tx and Rx LO. Through bias pads (BP, see inset) and Twave chokes the bias voltages $U_{\mathrm{Tx}, 0}$ and $U_{\mathrm{Rx}, 0}$ can be applied (output switch in position " 1 "). For transmission experiments, $U_{\mathrm{Tx}, 0}$ is modulated. In position " 0 " of the output switch (DC is zero for minimum noise) the signal current can be extracted via a transimpedance amplifier (TIA) and a lock-in amplifier (LIA), which is synchronized with the modulation of $U_{\mathrm{Tx}, 0}$. A gap of $1 \mu \mathrm{m}$ in the transmission line insulates the Tx and Rx biases. Insets: False-coloured magnified SEM images. [Fig. 3(a,b,c) modified from [8] (c) 2018 SNPG] 

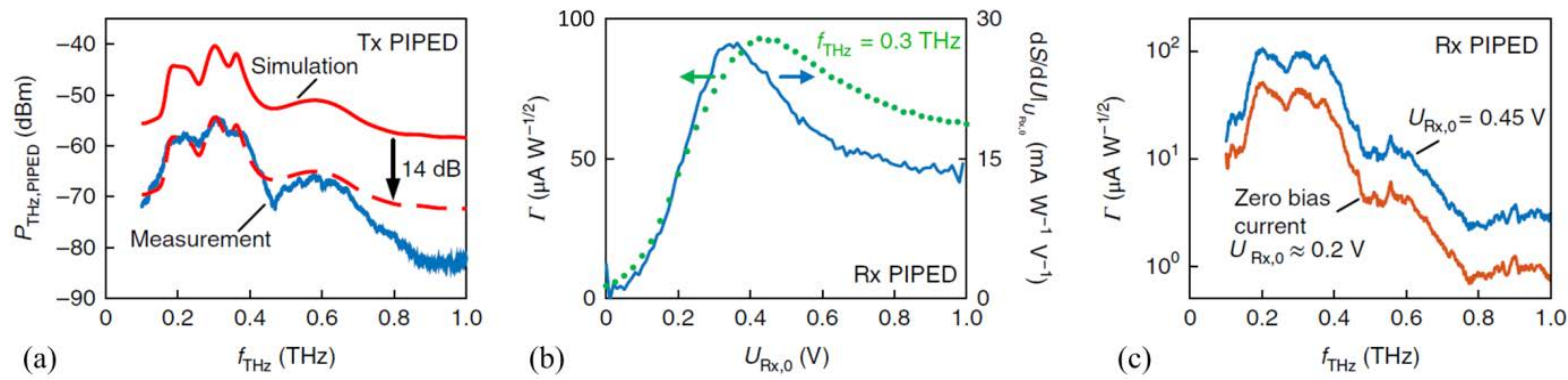

Fig. 4. Measured PIPED Tx and Rx performance. Dipole antenna and T-wave chokes are designed for a frequency of $0.385 \mathrm{THz}$. Peaks at lower frequencies are caused by resonances of the T-wave chokes. (a) Measured Tx T-wave power (blue) and simulated radiation (solid red) through the silicon substrate and an silicon lens. Simulated power levels are by $14 \mathrm{~dB}$ off due to inaccurately known coupling losses to the Rx. (b) T-wave Rx. Measured conversion factor $\Gamma=I_{\mathrm{BB}, 1} / \sqrt{P_{\mathrm{THz}}}$ (green, left axis) as a function of Rx bias voltage $U_{\mathrm{Rx}, 0}$ and biasdependent sensitivity slope $\mathrm{d} S / \mathrm{d} U @ U_{\mathrm{Rx}, 0}$ (blue, right axis) with a maximum near the carrier frequency $0.3 \mathrm{THz}$. (c) Rx conversion factor $\Gamma$ as a function of frequency both for zero-bias current (switch position " 0 " in Fig. 3 (c), $U_{\mathrm{Rx}, 0}=\varphi_{\mathrm{bi}} \approx 0.2 \mathrm{~V}$, red), and for a forward bias (maximum $\Gamma$ as in (b), $U_{\mathrm{Rx}, 0}=0.45 \mathrm{~V}$, blue). [Fig. 4(a,b,c) modified from [8] C $2018 \mathrm{SNPG]}$

to Eq. (2). The superimposed optical fields for $\mathrm{Tx}$ and $\mathrm{Rx}$ are denoted as $E_{\mathrm{Tx}, \mathrm{a}, \mathrm{b}}$ and $E_{\mathrm{Rx}, \mathrm{a}, \mathrm{b}}$, respectively. In Fig. 3(b), a scanning electron micrograph (SEM) is to be seen, where the PIPED is connected with its electrodes to the feed point of a dipole antenna. Silicon strip waveguides (red) provide the optical local oscillator power. A low-pass network (T-wave choke) decouples the bias voltage source from the T-wave circuits. The experimental set-up for on-chip transmission-line measurements is shown in Fig. 3(c). The Tx PIPED forward bias $U_{\mathrm{Tx}}=U_{\mathrm{Tx}, 0}+\hat{U}_{\mathrm{Tx}, 1} \cos \left(\omega_{\mathrm{mod}} t\right)$ is low-frequency modulated as in Fig. 2(c). At the end of the transmission line the homodyne Rx PIPED coherently detects the T-wave modulation with a lock-in amplifier. To reduce the noise, the Rx PIPED operates with zero-bias current (switch set to "0").

\section{MEASUREMENTS OF T-WAVE TRANSMITTER AND RECEIVER}

We measured the performance of PIPED Tx and Rx by generating and homodyne-receiving T-waves with frequencies $f_{\mathrm{THz}}$ up to $1.0 \mathrm{THz}$ [8], Fig. 4. Absolute power values are deduced from comparison to a commercial terahertz emitter (InGaAs pin photodiode, TOPTICA EK-000724). The measured radiated Tx power has a maximum of $-55 \mathrm{dBm}$ at a frequency near $f_{\mathrm{THz}}=0.3 \mathrm{THz}$, Fig. 4(a). Also the conversion factor $\Gamma=I_{\mathrm{BB}, 1} / \sqrt{P_{\mathrm{THz}}}=95 \mu \mathrm{A} \mathrm{W}^{-1 / 2}$ for the Rx peaks here, and so does the statically measured differential receiver sensitivity d $S /\left.\mathrm{d} U\right|_{U_{\mathrm{R} x, 0}}=28 \mathrm{~mA} /(\mathrm{W} \cdot \mathrm{V})$, Fig. 4(b),(c).

Because of the tiny PIPED dimensions, the Tx power and also the maximum $\mathrm{Rx}$ conversion gain $\frac{1}{2} \Gamma^{2} \times 50 \Omega=-66 \mathrm{~dB}$ are small compared to MMIC circuits with subharmonic mixers, but would be sufficient for many spectroscopic or diagnostic applications. For communication purposes, T-wave amplifiers and PIPED arrays, connected in parallel to the antenna feed point, could improve the sensitivity. When Tx and Rx are connected with the co-integrated transmission line, one could envisage to measure the absorption or the change of the refractive index induced by an applied liquid analyte as a function of the T-wave frequency.

\section{ACKNOWLEDGEMENTS}

We acknowledge support by the European Research Council (ERC Starting Grant 'EnTeraPIC', no. 280145; ERC Consolidator Grant 'TeraSHAPE', no. 773248), by the Alfried Krupp von Bohlen und Halbach Foundation, by the Helmholtz International Research School of Teratronics (HIRST), by the Karlsruhe School of Optics and Photonics (KSOP) and by the Karlsruhe Nano Micro Facility (KNMF).

\section{REFERENCES}

[1] S. Koenig, D. Lopez-Diaz, J. Antes, F. Boes, R. Henneberger, A. Leuther, A. Tessmann, R. Schmogrow, D. Hillerkuss, R. Palmer, T. Zwick, C. Koos, W. Freude, O. Ambacher, J. Leuthold, I. Kallfass: Wireless sub-THz communication system with high data rate. $\mathrm{Na}$ ture Photon. vol. 7, pp. 977-981, Oct. 2013.

[2] H. Ito, T. Furuta, Y. Muramoto, T. Ito, and T. Ishibashi: Photonic millimetre- and sub-millimetrewave generation using $\mathrm{J}$ band rectangular-waveguide-output uni-travelling-carrier photodiode module. Electron. Lett., vol. 42, pp. 1424-1425, Nov. 2006.

[3] H.-J. Song et al.: Uni-travelling-carrier photodiode module generating $300 \mathrm{GHz}$ power greater than $1 \mathrm{~mW}$. IEEE Microw. Wireless Compon. Lett., vol. 22, pp. 363-365, July 2012.

[4] D. Lopez-Diaz, I. Kallfass, A. Tessmann, A. Leuther, S. Wagner, M. Schlechtweg, O. Ambacher: A subharmonic chipset for gigabit communication around $240 \mathrm{GHz}$. IEEE Intern. Microw. Symp. Dig. (MTT-S), June 2012.

[5] D. Lopez-Diaz, S. Koenig, J. Antes, F. Boes, F. Kurz, R. Henneberger, A. Tessmann, A. Leuther, S. Wagner, M. Schlechtweg, O. Ambacher, I. Kallfass: A $240 \mathrm{GHz}$ quadrature receiver and transmitter for data transmission up to $40 \mathrm{Gbit} / \mathrm{s}$. Proc. $43^{\text {rd }}$ Europ. Microw. Integr. Circuits Conf., Oct. 2013. Paper EuMC/EuMIC06-4.

[6] S. Muehlbrandt, A. Melikyan, T. Harter, K. Köhnle, A. Muslija, P. Vincze, S. Wolf, P. Jakobs, Y. Fedoryshyn, W. Freude, J.
Leuthold, C. Koos, M. Kohl: Silicon-plasmonic internal-photoemission detector for $40 \mathrm{Gbit} / \mathrm{s}$ data reception. Optica, vol. 3, pp. 741-747, July 2016.

7] Freude, W.; Muehlbrandt, S.; Harter, T.; Melikyan, A.; Köhnle, K.; Muslija, A.; Vincze, P.; Wolf, S.; Jakobs, P.; Fedoryshyn, Y.; Leuthold, J.; Kohl, M.; Zwick, T.; Randel, S.; Koos, C.:'PIPED: A silicon-plasmonic high-speed photodetector. 19th Intern. Conf. on Transparent Optical Networks (ICTON'17), July 2017. Paper Mo.D4.1

[8] Harter, T.; Muehlbrandt, S.; Ummethala, S.; Schmid, A.; Nellen, S.; Hahn, L.; Freude, W.; Koos, C.: Silicon-plasmonic integrated circuits for terahertz signal generation and coherent detection. Nature Photon., vol. 12, pp. 625-633, Oct. 2018

[9] J. S. Helman, F. Sánchez-Sinencio: Theory of internal photoemission. Phys. Rev. B, vol. 7, pp. 3702-3706, April 1973.

[10] S. Zhu et al:: Low-cost and high-speed SOI waveguide-based silicide Schottky-barrier MSM photodetectors for broadband optical communications. IEEE Photon. Technol. Lett., vol. 20, pp. 13961398, Aug. 2008.

[11] B. Desiatov et al.: Plasmonic enhanced silicon pyramids for internal photoemission Schottky detectors in the near-infrared regime. Optica, vol. 2, pp. 335-338, April 2015.

[12] S. M. Sze: Physics of semiconductor devices, 2nd Ed. New York: John Wiley \& Sons, 1981. 